\title{
Interband optical transitions in DNA-like systems
}

\author{
E. Díaz, A. V. Malyshev, ${ }^{*}$ and F. Domínguez-Adame \\ GISC, Departamento de Física de Materiales, Universidad Complutense, E-28040 Madrid, Spain \\ (Received 12 December 2006; revised manuscript received 9 July 2007; published 26 November 2007)
}

\begin{abstract}
The underlying band structure of the uniform DNA suggests the allowance of interband optical transitions. We consider such transitions in the uniform synthetic DNA, such as the $\operatorname{poly}(G)$ - $\operatorname{poly}(C)$ DNA, within a simple tight-binding model with a minimum set of parameters. We demonstrate that the helical conformation of the DNA strands results in unusual interband optical transitions all of which appear to be indirect in $k$ space although the system has a direct band gap energy structure. Simple relationships of the optical gap and absorption linewidth to tight-binding model parameters are found. We study also the effect of disorder in base levels (relevant for the wet form of the DNA) on interband optical transitions.
\end{abstract}

DOI: 10.1103/PhysRevB.76.205117

PACS number(s): 87.14.Gg

\section{INTRODUCTION}

Controlled manipulation of single DNA molecules have enabled direct measurements of electrical transport through the DNA. ${ }^{1}$ In addition to its size in the nanometer scale range, the DNA assembles spontaneously. ${ }^{2}$ It is believed therefore to be a promising candidate for designing new nanostructures. ${ }^{3}$ The possibility to use the DNA as a programmable material for nanotechnology applications have sparked intensive research in the field (see Ref. 4, and references therein for an overview).

Experiments on electrical transport through dry and wet DNA molecules revealed a variety of results: DNA has been reported to demonstrate proximity-induced superconducting, ${ }^{5}$ metallic, ${ }^{6-9}$ semiconducting,, $10-13$ and insulating ${ }^{14,15}$ behavior. The observed differences are believed to be related to contact effects, the environment of the DNA molecule and the sequence of nucleotides.

Semiconducting behavior of dry uniform DNA, such as the $\operatorname{poly}(G)$-poly $(C)$, was established experimentally. ${ }^{1}$ Much earlier, it was predicted by theoretical calculations. ${ }^{16}$ Effective Hamiltonian models, based on the tight-binding approximation, ${ }^{17-27}$ provide a reasonable description of the semiconductor gap observed in experiments. In this contribution we point out that the underlying electronic band structure with full valence band and empty conduction band suggests that interband optical transitions can occur in DNA. We use the tight-binding ladder ${ }^{17}$ model with a minimal set of adjustable parameters to describe the main features of the optically induced electronic transitions in the synthetic semiconducting DNA. We study the relationship of the features of the absorption spectra to model parameters. We focus also on the effect of disorder on the optical transitions in DNA.

The paper is organized as follows. In Sec. II we describe the tight-binding model that we use for the $\operatorname{poly}(G)$-poly $(C)$ DNA. Electronic states and band structure of the DNA are discussed in Sec. III and interband optical transitions in periodic DNA are studied in Sec. IV. Effects of disorder in bases molecules (which is relevant for wet DNA) are analyzed in detail in Sec. V. The impact of the helix geometry of the DNA molecule on the interband optical transitions is studied in Sec. VI. Finally, in Sec. VII we summarize the results and their relevance for experimental measurements necessary to infer the main characteristics of the interband optical transitions.

\section{LADDER MODEL OF DNA-LIKE SYSTEMS}

Our analysis proceeds as follows. We consider the ladder model $^{17}$ of the DNA based on a tight-binding Hamiltonian with few adjustable parameters. Similar models have been previously introduced by several authors ${ }^{18-27}$ to explain the main semiconducting features observed in current-voltage characteristics in dry synthetic $\operatorname{poly}(G)$-poly $(C)$ DNA. ${ }^{1}$ Intramolecule transitions are beyond the scope of this work, and consequently only a single molecular orbital is assigned to each base. Hereafter the nearest-neighbor approximation is used for interstrand and intrastrand hoppings, in order to describe the $\pi$ stack of base pairs. We assume that values of interbase hopping parameters depend on the bases, describing it by the hoppings $t_{G G}, t_{C C}$, and $t_{\perp}$ (see Fig. 1).

Because the backbone is believed to have little effect as a transmission channel in dry DNA, ${ }^{25}$ it is neglected completely within the framework of this model. This is the case when the electronic bands arising from the hybridization of backbone levels lie at a much higher energy than the levels of the bases. ${ }^{28}$

Once the model has been established, we can write down the equation for the electronic amplitudes at the bases. Let us denote these amplitudes as $\psi_{n}^{s}$, where $n=1,2, \ldots, N$ labels a

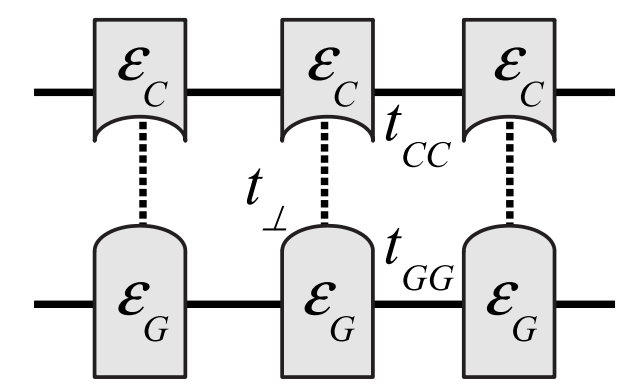

FIG. 1. Schematic view of a fragment of $\operatorname{poly}(G)$-poly $(C)$ DNA molecules, excluding the sugar-phosphate backbone. Only three different hoppings are considered: the interstrand $\left(t_{\perp}\right)$ and the intrastrand ones $t_{G G}$ and $t_{C C}$. 


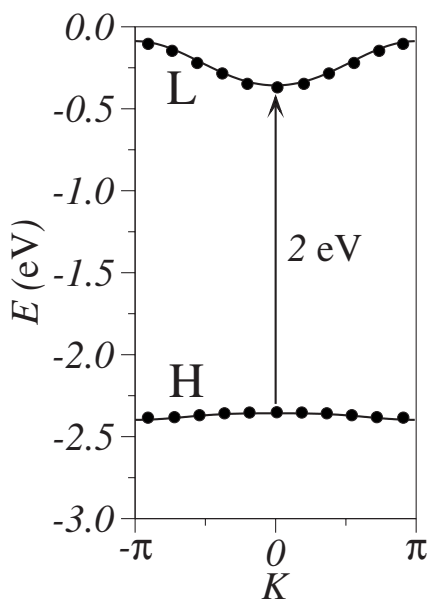

FIG. 2. HOMO $(H)$ and LUMO $(L)$ bands in poly $(G)$-poly $(C)$ DNA. Solid lines correspond to Eq. (2) within the ladder model and solid circles are the energy values taken from Ref. 29.

base pair and the superscript $s=G, C$ labels the two strands. The equations for the amplitudes are then given by

$$
\begin{aligned}
& \left(E-\varepsilon_{C}\right) \psi_{n}^{C}=t_{C C}\left(\psi_{n+1}^{C}+\psi_{n-1}^{C}\right)+t_{\perp} \psi_{n}^{G}, \\
& \left(E-\varepsilon_{G}\right) \psi_{n}^{G}=t_{G G}\left(\psi_{n+1}^{G}+\psi_{n-1}^{G}\right)+t_{\perp} \psi_{n}^{C} .
\end{aligned}
$$

\section{ELECTRONIC BAND STRUCTURE}

In this section we consider a uniform DNA molecule, in which all base energies along each strand take the same value. Solution of Eq. (1) with rigid boundary conditions are easily found since they correspond to two coupled quantum wires; closed expression for the dispersion relation can also be obtained analytically:

$$
E(K)=\bar{E}_{+}(K) \pm \sqrt{\bar{E}_{-}^{2}(K)+t_{\perp}^{2}} .
$$

For brevity we have now defined

$$
\bar{E}_{ \pm}(K)=\frac{1}{2}\left[E_{G}(K) \pm E_{C}(K)\right]
$$

where $K=\pi k /(N+1)$ with $k=1,2, \ldots, N$. Here $E_{G}(K)=\varepsilon_{G}$ $+2 t_{G G} \cos (K)$ and $E_{C}(K)=\varepsilon_{C}+2 t_{C C} \cos (K)$ are the dispersion relationships in each strand.

Parameters for the ladder model are chosen to fit $a b$ initio band structure calculations. To this end, we compare density functional calculations for the uniform $\operatorname{poly}(G)$-poly $(C)$ DNA (Ref. 29) and the band structure obtained from Eq. (2). The best fitting parameters are $\varepsilon_{C}=-0.75 \mathrm{eV}, \varepsilon_{G}$ $=-1.85 \mathrm{eV}, t_{C C}=-0.105 \mathrm{eV}, t_{G G}=0.047 \mathrm{eV}$, and $t_{\perp}=0.917$ $\mathrm{eV}$. Figure 2 shows the calculated band structure. In this case hoppings along the two strands $t_{C C}$ and $t_{G G}$ have opposite signs which results in a direct band gap energy structure. We note here that parameter sets which are traditionally used for transport calculations (see Ref. 25, and references therein) suggest equal sign of intrastrand hoppings $t_{C C}$ and $t_{G G}$. The latter choice, making no difference for transport, would lead to the indirect band gap electronic structure which would have strong impact on optical properties, e.g., the optical gap. Therefore, comparison of transport and optical measurements can shed light on this underlying issue.

Moreover, the occurrence of a large inter-strand hopping in this model is a consequence of the huge gap obtained by density functional calculations from Ref. 29 and seen in Fig. 2. Some authors claim that Coulomb interaction is crucial for obtaining such a large gap. ${ }^{30}$ This interaction may cause a large interstrand hopping in an effective, one-body model such as the present one.

\section{INTERBAND OPTICAL TRANSITIONS}

The solutions presented in the previous section will be the starting point to calculate the optical absorption coefficient due to interband transitions in DNA molecules. To simplify the calculations, we will restrict ourselves to zero temperature and assume that the lower (HOMO) band is full and the upper (LUMO) band is empty. Under these assumptions, the absorption coefficient due to interband transition is given by the Fermi golden rule as

$$
A(\hbar \omega) \propto \sum_{i} \sum_{f}|\langle i|\hat{e} \cdot \vec{r}| f\rangle|^{2} \delta\left(E_{f}-E_{i}-\hbar \omega\right),
$$

where $i$ and $f$ run over the states of the HOMO and LUMO band, respectively. Here $\hat{e}$ is the unit vector along the polarization direction of the incoming electromagnetic plane wave. We then focus on the square of the optical transition matrix elements $F_{i j}=|\langle i|\hat{e} \cdot \vec{r}| f\rangle|^{2}$. The $\delta$ function in Eq. (3) is replaced by a square-box function of width $5 \mathrm{meV}$ to take into acount the homogeneous broadening.

The intrastrand hopping parameters that we obtained in Sec. III have opposite signs for the two strands, which leads to the energy structure with a direct band gap at $\Gamma$ point $(K=0)$. The minimum energy difference between the two bands (determining the electronic gap) is also at the $\Gamma$ point, suggesting that the optical and electronic gaps coincide. Figure 3 shows the absorption spectra for parallel (upper panel) and perpendicular (lower panel) polarizations. The optical gap is about $2.0 \mathrm{eV}$ in both cases; it corresponds to transitions at the $\Gamma$ point and coincides with the electronic gap (see Fig. 2).

\section{EFFECTS OF DISORDER}

In this section we consider the effects of the environment on the interband optical transitions described above. Disorder can originate from interactions with a random environment of solute molecules and ions surrounding the DNA molecule which can affect not only the backbone but also the base molecules. We account for these interactions by considering random base energies uniformly distributed within a box of width $w$ and centered around $\varepsilon_{G}$ or $\varepsilon_{C}$, depending on the strand. We restrict ourselves to static disorder as we consider the $T=0$ case and the molecule is supposed to be embedded into a glassy host.

Figure 4 shows the absorption spectra for synthetic $\operatorname{poly}(G)-\operatorname{poly}(C)$ DNA with disorder in the base energies. 


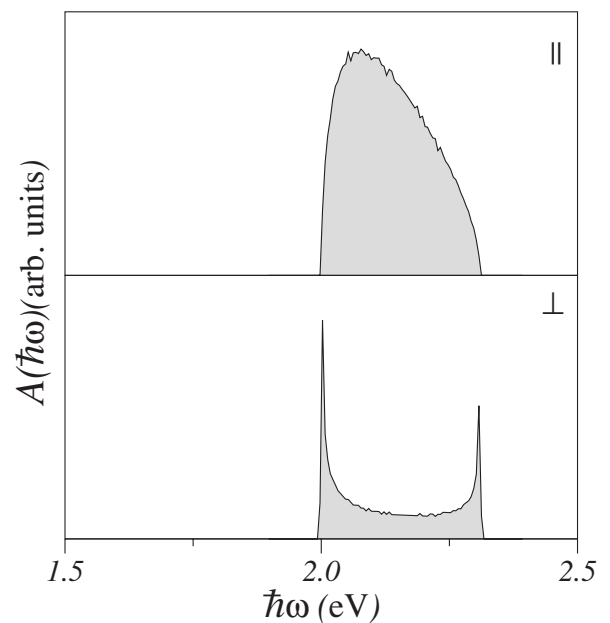

FIG. 3. Absorption spectra for parallel (upper panel) and perpendicular (lower panel) polarizations for $N=1000$ and parameter set obtained in Sec. III. In both cases the optical gap of $2.0 \mathrm{eV}$ is equal to the electronic gap.

The results demonstrate that the absorption band edge remains almost unchanged for perpendicularly polarized light. On the contrary, the absorption spectrum for parallel polarization is sensitive to the magnitude of the disorder. At moderate disorder $(w=0.75 \mathrm{eV})$ the electronic gap is still open and the corresponding absorption spectrum broadens as compared to the case of the homogeneous DNA discussed in the previous section. As the magnitude of disorder increases $(w$ $=1.25 \mathrm{eV}$ ) the electronic gap closes, as the left panel of Fig. 4 shows.

\section{IMPACT OF THE HELIX CONFORMATION OF THE DNA STRANDS}

The considered model neglect the double helix geometry of the DNA molecule. However, the spatial arrangement of
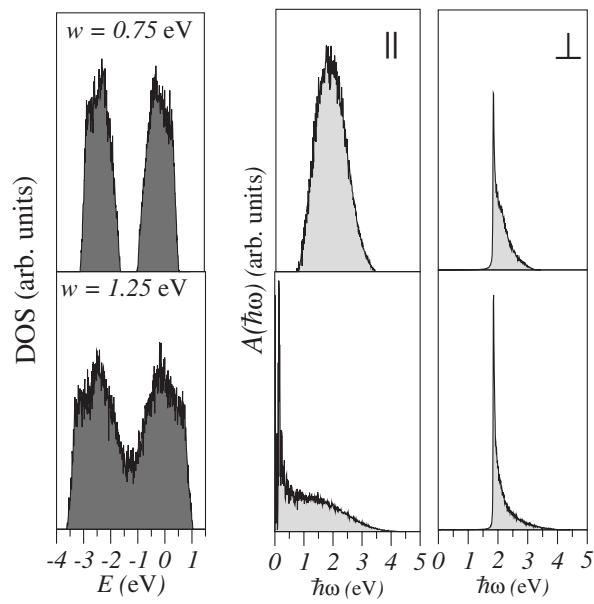

FIG. 4. Left panels show the DOS for two different magnitudes of disorder in base energies indicated in each plot. The corresponding absorption spectra for parallel (middle panels) and perpendicular (right panels) polarizations are also shown. The curves $A(\hbar \omega)$ comprise averaging over 1000 realizations of base disorder with the bin of $5 \mathrm{meV}$. bases has recently been demonstrated to be crucial for transport properties in the presence of the perpendicular electric field. ${ }^{27}$ It can also be relevant when dealing with optical dipole moments of the electronic transitions. To improve the analysis, we consider the double helix structure of DNA molecules: the coordinates of the bases along both strands can be set as

$$
\begin{gathered}
x_{n}^{s}=R \cos \left(\frac{2 \pi}{T} n+\beta_{s} \phi_{0}\right), \\
y_{n}^{s}=R \sin \left(\frac{2 \pi}{T} n+\beta_{s} \phi_{0}\right), \\
z_{n}^{s}=v n,
\end{gathered}
$$

where $n=1, \ldots, N$ and $s=C, G$. Here $\beta_{C}=+1$ and $\beta_{G}=-1$. We consider the $B$ form of the DNA with the following parameters of the double helix: full twist period of 10 base pairs, i.e., $T=10$. Finally, we set $\phi_{0}=\pi / 3$, so that the relative phase difference between the helices is $2 \phi_{0}=2 \pi / 3$, and the minor groove is one half of the major one.

The helical conformation of the DNA strands results in unusual selection rules for inter-band optical transitions in the case of perpendicular polarization. Assume for simplicity that $\varepsilon_{G}=\varepsilon_{C}$. In this simple case the wave functions have well-defined symmetries not only along the base-stacking direction and also in the transversal direction. These symmetries give rise to selection rules for the electron momentum (not shown here). It is then a matter of simple algebra to demonstrate that

$$
F_{i f} \propto \frac{\sin ^{2}\left(\left|K_{i} \pm K_{f}\right|-2 \pi / T\right)}{\left(\left|K_{i} \pm K_{f}\right|-2 \pi / T\right)^{2}}=\pi \delta\left(\left|K_{i} \pm K_{f}\right|-2 \pi / T\right) .
$$

Thus, the harmonic modulation of dipole moments brings in the additional effective momentum $2 \pi / T$, which changes the selection rule from that conserving the true total momentum to the conservation of the sum of the total and the effective momenta. As the result the strongest transitions appear to be indirect in $k$ space. Changes of the absorption band that result from the modification of the selection rule depend on the energy dispersion of the bands. For the direct band gap case the absorption band narrows with respect to the planar case. The amount of shrinking depends on the model parameters: the smaller are the effective masses at the bottoms of the bands the larger is the effect.

The absorption coefficient of the interband optical transitions in the twisted model is presented in Fig. 5 for perpendicular polarization (the spectrum for parallel polarization remains unchanged). The height of the absorption spectrum is reduced as compared to the planar case. The reduction is due to the fact that dipole moments are rotating around the molecule axis, so roughly half of them are perpendicular to the field and therefore do not interact with it. Because the energy structure has direct band gap for the chosen parameters, the helical spectrum is narrower than the planar one. However, the narrowing is very small because effective masses are large (small dispersion). 


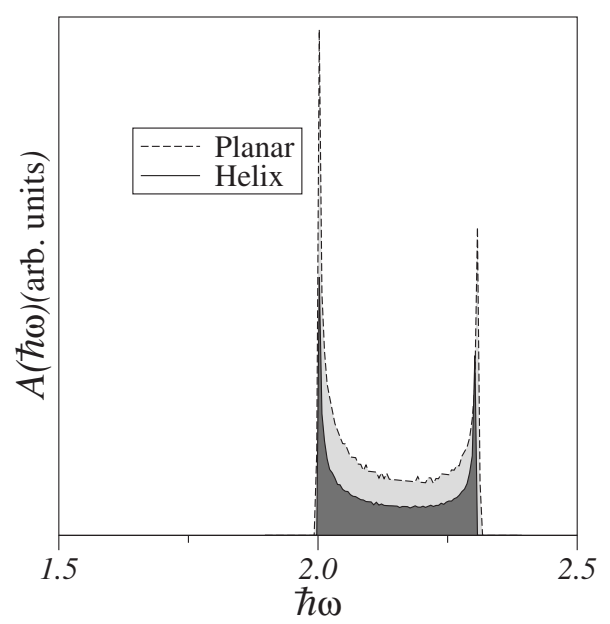

FIG. 5. Absorption spectra for perpendicular polarizations within the planar ladder (dashed line) and the twisted ladder (solid line) models. The number of bases at each strand is $N=1000$.

We note finally that parallel and perpendicular absorption spectra could possibly be measured in self-assembled monolayer of DNA on gold substrates, ${ }^{31-35}$ in which DNA molecules have well-defined orientation.

\section{CONCLUSIONS}

We considered interband optical transitions in the semiconducting synthetic DNA, such as the $\operatorname{poly}(G)$-poly $(C)$ DNA. To study the optical properties we used a tight-binding model with a minimal parameter sets. By fitting the band structure, that was calculated by $a b$ initio methods, ${ }^{29}$ we have obtained a parameter set in which hoppings along each strand have opposite signs. The latter leads to a direct band gap energy structure with equal optical and electronic gaps. We put forward a simple relationship of the optical gap to tight-binding model parameters and argue that combined optical and electric measurements can provide an insight into the underlying electronic band structure of the DNA.

To address optical properties of the DNA in its wet form, we have studied the effect of static disorder in the base levels on interband optical transitions. Static disorder reduces both optical and electronic gaps. The closure of the electronic gap by the disorder provides, in particular, a simple explanation of the observed ohmic behavior of DNA conductivity in disordered aqueous environment. ${ }^{9}$

We show also that the helical confirmation of the DNA strands result in unusual selection rules for interband optical transitions in the case of perpendicular polarization of incoming light. Such transitions appear to be indirect in momentum space. On these grounds, we demonstrate that analysis of absorption spectra for parallel and perpendicular polarizations can provide valuable information on details of the DNA energy structure.

Although matrix elements of intramolecule excitations are several orders of magnitude larger than those involving molecular levels of different bases, ${ }^{36,37}$ the intramolecule transition energy of about $3.7 \mathrm{eV}^{37}$ observed in wet DNA is probably well above the absorption band edge for interband transitions. Thus, low-energy features in the absorption spectra due to interband transitions are relatively weak but well separated from the strong intramolecule excitations, which suggests that they can be observed, although this could be challenging from the experimental point of view.

Finally, it is worth mentioning that the model we use does not account for thermally activated hopping or polaronic effects. ${ }^{10}$ We believe that such mechanisms should also be considered when interpreting experimental spectra. Work along these lines is in progress.

\section{ACKNOWLEDGMENTS}

The authors thank E. Maciá, A. Rodríguez, and P. A. Orellana for helpful discussions. This work was supported by MEC (Project MOSAICO). A.V.M. acknowledges financial support from MEC through the Ramón y Cajal program.
*On leave from Ioffe Physico-Technical Institute, Saint Petersburg, Russia.

${ }^{1}$ D. Porath, A. Bezryadin, S. de Vries, and C. Dekker, Nature (London) 403, 635 (2000).

${ }^{2}$ H. Yan, S. H. Park, G. Finkelstein, J. H. Reif, and T. H. LaBean, Science 301, 1882 (2003).

${ }^{3}$ N. C. Seeman, Nature (London) 421, 427 (2003).

${ }^{4}$ Introducing Molecular Electronics, edited by G. Cuniberti, G. Fagas, and K. Richter (Springer, Berlin, 2005).

${ }^{5}$ A. Yu. Kasumov, M. Kociak, S. Guéron, B. Reulet, V. T. Volkov, D. V. Klinov, and H. Bouchiat, Science 291, 280 (2001).

${ }^{6}$ Y. Okahata, T. Kobayashi, K. Tanaka, and M. J. Shimomura, J. Am. Chem. Soc. 120, 6165 (1998).

${ }^{7}$ H. W. Fink and C. Schönenberger, Nature (London) 398, 407 (1999).

${ }^{8}$ A. Rakitin, P. Aich, C. Papadopoulos, Yu. Kobzar, A. S. Vede- neev, J. S. Lee, and J. M. Xu, Phys. Rev. Lett. 86, 3670 (2001). ${ }^{9}$ O. Legrand, D. Côte, and U. Bockelmann, Phys. Rev. E 73, 031925 (2006).

${ }^{10}$ K.-H. Yoo, D. H. Ha, J.-O. Lee, J. W. Park, J. Kim, J. J. Kim, H.-Y. Lee, T. Kawai, and H. Y. Choi, Phys. Rev. Lett. 87, 198102 (2001).

${ }^{11}$ J. S. Hwang, K. J. Kong, D. Ahn, G. S. Lee, D. J. Ahn, and S. W. Hwang, Appl. Phys. Lett. 81, 1134 (2002).

${ }^{12}$ B. Q. Xu, P. M. Zhang, X. L. Li, and N. J. Tao, Nano Lett. 4, 1105 (2004).

${ }^{13}$ H. Cohen, C. Nogues, R. Naaman, and D. Porath, Proc. Natl. Acad. Sci. U.S.A. 102, 11589 (2005).

${ }^{14}$ E. Braun, Y. Eichen, U. Sivan, and G. Ben-Yoseph, Nature (London) 391, 775 (1998).

${ }^{15}$ A. J. Storm, J. van Noort, S. de Vries, and C. Dekker, Appl. Phys. Lett. 79, 3881 (2001). 
${ }^{16}$ P.-O. Löwdin, Rev. Mod. Phys. 35, 724 (1963).

${ }^{17}$ K. Iguchi, Int. J. Mod. Phys. B 11, 2405 (1997).

${ }^{18}$ K. Iguchi, J. Phys. Soc. Jpn. 70, 593 (2001).

${ }^{19}$ G. Cuniberti, L. Craco, D. Porath, and C. Dekker, Phys. Rev. B 65, 241314(R) (2002).

${ }^{20}$ S. Roche, D. Bicout, E. Maciá, and E. Kats, Phys. Rev. Lett. 91, 228101 (2003).

${ }^{21}$ K. Iguchi, Int. J. Mod. Phys. B 18, 1845 (2004).

${ }^{22}$ H. Yamada, Phys. Lett. A 332, 65 (2004); Int. J. Mod. Phys. B 18, 1697 (2004); Phys. Rev. B 69, 014205 (2004).

${ }^{23}$ V. M. Apalkov and T. Chakraborty, Phys. Rev. B 71, 033102 (2005).

${ }^{24}$ H. Mehrez and M. P. Anantram, Phys. Rev. B 71, 115405 (2005).

${ }^{25}$ D. Klotsa, R. A. Römer, and M. S. Turner, Biophys. J. 89, 2187 (2005).

${ }^{26}$ A. Rodríguez, R. A. Römer, and M. S. Turner, Phys. Status Solidi B 243, 373 (2006).

${ }^{27}$ A. V. Malyshev, Phys. Rev. Lett. 98, 096801 (2007).

${ }^{28}$ E. Maciá and S. Roche, Nanotechnology 71, 3002 (2006).
${ }^{29}$ E. Artacho, M. Machado, D. Sánchez-Portal, P. Ordejón, and J. M. Soler, Mol. Phys. 101, 1587 (2003).

${ }^{30}$ J. Yi, Phys. Rev. B 68, 193103 (2003).

${ }^{31}$ S. O. Kelley, J. K. Barton, N. M. Jackson, and M. G. Hill, Bioconjugate Chem. 8, 31 (1997).

${ }^{32}$ S. O. Kelley, J. K. Barton, N. M. Jackson, L. D. McPherson, A. B. Potter, E. M. Spain, M. J. Allen, and M. G. Hill, Langmuir 14, 6781 (1998).

${ }^{33}$ S. O. Kelley, N. M. Jackson, M. G. Hill, and J. K. Barton, Angew. Chem., Int. Ed. 38, 941 (1999).

${ }^{34}$ G. Hartwich, D. J. Caruana, T. de Lumley-Woodyear, Y. B. Wu, C. N. Campbell, and A. Heller, J. Am. Chem. Soc. 121, 10803 (1999).

${ }^{35}$ M. Sam, E. M. Boon, J. K. Barton, M. G. Hill, and E. M. Spain, Langmuir 17, 5727 (2001).

${ }^{36}$ E. Helgren, G. Grüner, A. Omerzu, D. Mihailovic, R. Podgornik, and H. Grimm, arXiv:cond-mat/0111299 (unpublished).

${ }^{37}$ A. Hubsch, R. G. Endres, D. L. Cox, and R. R. P. Singh, Phys. Rev. Lett. 94, 178102 (2005). 